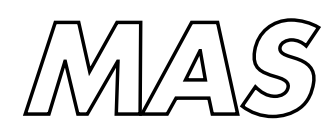

Modelling, Analysis and Simulation

Modelling, Analysis and Simulation
MAS $\begin{gathered}\text { Computation of 3D Steady Navier-Stokes Flow with } \\ \text { Free-Surface Gravity Waves }\end{gathered}$

M.R. Lewis, B. Koren, H.C. Raven

Report MAS-E0324 December 16, 2003 
CWI is the National Research Institute for Mathematics and Computer Science. It is sponsored by the Netherlands Organization for Scientific Research (NWO).

CWI is a founding member of ERCIM, the European Research Consortium for Informatics and Mathematics.

CWI's research has a theme-oriented structure and is grouped into four clusters. Listed below are the names of the clusters and in parentheses their acronyms.

Probability, Networks and Algorithms (PNA)

Software Engineering (SEN)

Modelling, Analysis and Simulation (MAS)

Information Systems (INS)

Copyright (C) 2003, Stichting Centrum voor Wiskunde en Informatica

P.O. Box 94079, 1090 GB Amsterdam (NL)

Kruislaan 413, 1098 SJ Amsterdam (NL)

Telephone +31205929333

Telefax +31205924199

ISSN 1386-3703 


\title{
Computation of 3D Steady Navier-Stokes Flow with Free-Surface Gravity Waves
}

\begin{abstract}
In this paper an iterative method for the computation of stationary gravity-wave solutions is investigated, using a novel formulation of the free-surface (FS) boundary-value problem. This method requires the solution of a sequence of stationary Reynolds-Averaged Navier-Stokes subproblems employing the so-called quasi free-surface condition. The numerical performance of this new approach is investigated for two test cases. The first test case involves the computation of the 3D gravity-wave pattern due to a pressure-perturbation imposed on a uniform flow. The second is the gravity-wave pattern generated by a realistic ship-hull form, known as Series 60. Results of the ship-hull cases are compared with experimental data.
\end{abstract}

2000 Mathematics Subject Classification: 76-xx

Keywords and Phrases: incompressible Navier-Stokes equations; free-surface waves 


\title{
Computation of 3D Steady Navier-Stokes Flow with Free-Surface Gravity Waves
}

\author{
Mervyn R. Lewis ${ }^{1}$, Barry Koren ${ }^{1}$, and Hoyte C. Raven ${ }^{2}$ \\ 1 CWI, P.O. Box 94079, 1090 GB Amsterdam, The Netherlands \\ 2 MARIN, P.O. Box 28, 6700 AA Wageningen, The Netherlands
}

\begin{abstract}
In this paper an iterative method for the computation of stationary gravitywave solutions is investigated, using a novel formulation of the free-surface (FS) boundaryvalue problem. This method requires the solution of a sequence of stationary ReynoldsAveraged Navier-Stokes subproblems employing the so-called quasi free-surface condition. The numerical performance of this new approach is investigated for two test cases. The first test case involves the computation of the 3D gravity-wave pattern due to a pressure perturbation imposed on a uniform flow. The second is the computation of the gravity-wave pattern generated by a realistic ship-hull form, known as Series 60 . Results of the ship-hull case are compared with experimental data.
\end{abstract}

\section{Introduction}

In fluid dynamics a large class of problems exists in which a free surface is present. The inherent difficulty of this class of problems is the interdependence of the free surface (FS) location and the unknowns of the bulk-flow problem. Examples of FS flow problems in the physical sciences and engineering are vast. Here we consider the stationary gravity waves generated by ships moving with constant speed. Although much of this wave system is adequately described by inviscid theory, certain areas in the ship-hydrodynamics problem, such as the boundary layer and the wake, require a viscous description. Therefore, present-day developments are primarily directed towards computing stationary FS Navier-Stokes flow problems. Current numerical methods for solving the stationary viscous FS flow problem often employ a time-dependent formulation and integrate until a steady state is reached. This approach typically displays two defects, viz., high computational costs due to persistent transient behaviour of the gravity waves and substantial spatial damping of these waves. To reduce the computational effort of solving FS Navier-Stokes flow an efficient iterative method, employing a novel formulation of the FS flow problem was introduced in [1]. This method employs the so-called quasi free-surface condition (QFSC). The method shows mesh-independent asymptotic convergence rates for a $2 \mathrm{D}$ test case.

The emphasis of this paper lies on the extension to 3D of this method and on the investigation of its convergence behaviour. The numerical method is applied to two 3D test cases. The first test case concerns the computation of the wave system due to a pressure perturbation imposed on the FS of a uniform flow. In the second test case the wave pattern is computed around the Series 60 shiphull form, a benchmark problem for FS methods in the ship-hydrodynamics community. 


\section{Mathematical Model}

In this section a short description is given of the mathematical model which is involved and, secondly, a short summary of the free surface conditions from which the QFSC can be derived.

Let $\Omega(\boldsymbol{x}) \subset R^{3}$ denote the physical domain which is occupied by the fluid and $\partial \Omega(\boldsymbol{x})=\Gamma_{\mathrm{FS}} \cup \Gamma_{0}$ the boundary of $\Omega(\boldsymbol{x})$, where $\Gamma_{\mathrm{FS}}$ denotes the FS and $\Gamma_{0}$ the remaining (fixed) part of the boundary. The dynamics of the viscous, incompressible flow subjected to a constant gravitational force is described by the Navier-Stokes equations, which read

$$
\begin{aligned}
\nabla \cdot \boldsymbol{u} \boldsymbol{u}^{T}+\nabla \varphi-\nabla \cdot \boldsymbol{\tau}(\boldsymbol{u})=0, & \forall \boldsymbol{x} \in \Omega, \\
\nabla \cdot \boldsymbol{u}=0, & \forall \boldsymbol{x} \in \Omega,
\end{aligned}
$$

where $\boldsymbol{\tau}(\boldsymbol{u})$ is the viscous stress tensor and $\nabla \varphi(\boldsymbol{x})=\nabla p(\boldsymbol{x})+\mathrm{Fr}^{-2} \boldsymbol{e}_{z}$ the gradient of the hydrodynamic pressure containing the Froude number $\mathrm{Fr}=U_{\infty} / \sqrt{g \ell}$ with $g$ the acceleration of gravity and $\ell$ the reference length.

The dynamics of the free surface boundary, $\Gamma_{\mathrm{FS}}$, is governed by the following conditions. Assuming that the FS can be described as a single-valued function, denoted by $\Gamma_{\mathrm{FS}}=\{(\boldsymbol{x}): z=\zeta(x, y)\}$, the kinematic condition can be written as

$$
\boldsymbol{u} \cdot \nabla \zeta(x, y)=\boldsymbol{u} \cdot \boldsymbol{e}_{z}, \quad \forall \boldsymbol{x} \in \Gamma_{\mathrm{FS}},
$$

where $\boldsymbol{e}_{z}$ is the unit vector directed in the opposite direction of the gravitational force. The FS has to adhere to the three dynamic conditions imposed by the assumption of vanishing interfacial stresses. The dynamic condition in the direction normal to the FS reads

$$
p(\boldsymbol{x})-2 \operatorname{Re}^{-1} \frac{\partial \boldsymbol{u}_{n}}{\partial \boldsymbol{n}}=p_{\mathrm{FS}}(\boldsymbol{x}),
$$

whereas the conditions in the two tangential directions are

$$
\boldsymbol{t}^{(\alpha)} \cdot \boldsymbol{\tau}(\boldsymbol{u}) \cdot \boldsymbol{n}=0, \quad \alpha=1,2 .
$$

Here $p_{\mathrm{FS}}(\boldsymbol{x})$ is the specified pressure distribution along the FS. In our first and second test case, $p_{\mathrm{FS}}(\boldsymbol{x}) \neq 0$ and $p_{\mathrm{FS}}(\boldsymbol{x})=0$, respectively. For the practical application envisaged here the viscous contribution in (3) will be neglected. Also, the effects of surface tension can be safely ignored.

Many modern FS-iteration methods apply a formulation in which they solve (1a), (1b), in time-dependent form, subjected to the normal dynamic condition applied at an approximate location of the FS, followed by an update of the FS using the kinematic condition in time-dependent form. This approach is disadvantageous because it decouples the two FS conditions. It is the combination of the kinematic and (normal) dynamic condition which is responsible for wave-like solutions. Therefore it was proposed in [1] to combine these conditions resulting in the quasi free-surface condition (QFSC)

$$
\operatorname{Fr}^{2} \boldsymbol{u} \cdot \nabla \varphi-\boldsymbol{u} \cdot \boldsymbol{e}_{z}=\mathrm{Fr}^{2} \boldsymbol{u} \cdot \nabla p_{\mathrm{FS}}(\boldsymbol{x}), \quad \forall \boldsymbol{x} \in \Gamma_{\mathrm{FS}} .
$$

Note the nonlinearity of the boundary condition. 


\section{Computational Method}

It has been shown that the time-integration method to solve the steady-state FS flow problem is computationally inefficient. This is due to the fact that the attenuation of the surface-gravity waves behaves like $\mathcal{O}\left(t^{(1-d) / 2}\right)$ in $R^{d}$, see e.g. $[1,2]$. To circumvent this slow transient behaviour we adopt an iterative method for the stationary formulation of the FS flow problem. This iterative method requires the solution of a number of stationary Navier-Stokes subproblems employing the QFSC. The solution of the FS flow problem can be found by iterating the following two steps:

1. For a given boundary $\Gamma_{\mathrm{FS}}$, solve $(\boldsymbol{u}(\boldsymbol{x}), \varphi(\boldsymbol{x}))$ from (1a), (1b) with on $\Gamma_{\mathrm{FS}}$ the boundary conditions (4), (5) and on $\Gamma_{0}$ other appropriate boundary conditions.

If $\left\|p-p_{\mathrm{FS}}\right\|$ is still larger than a chosen small tolerance (assume that at convergence $p=p_{\mathrm{FS}}$ ), then do step 2, else stop.

2. Use the solution $(\boldsymbol{u}(\boldsymbol{x}), \varphi(\boldsymbol{x}))$ of step 1 to obtain a (better) approximation of $\Gamma_{\mathrm{FS}}$ according to

$$
\left\{\left(x, y, z=\operatorname{Fr}^{2}\left(\varphi(\boldsymbol{x})-p_{\mathrm{FS}}(\boldsymbol{x})\right):(x, y, z) \in \Gamma_{\mathrm{FS}}\right\}\right.
$$

next return to step 1 .

The nonlinear stationary Navier-Stokes subproblem is discretized by replacing the differential operators by second and third-order finite-difference schemes and next solved by Newton's method. The resulting linear system of algebraic equations is solved using a space-marching strategy whereby the marching is performed in the main flow direction. As a result a number of smaller systems has to be solved, which is done by preconditioned GMRES. More details on this solution procedure can be found in [3].

\section{$4 \quad$ Numerical Results}

\subsection{Pressure Perturbation}

The first test case considers the computation of the gravity-wave pattern due to a pressure perturbation imposed on a uniform flow. The perturbation has the Gaussian distribution

$$
p_{\mathrm{FS}}(x, y)=P e^{-\alpha r^{2}}, \quad r^{2}=\left(x-x_{0}\right)^{2}+\left(y-y_{0}\right)^{2}, \quad \forall(x, y) \in \Gamma_{\mathrm{FS}}
$$

where the parameter values $P=0.05, \alpha=-4$ and $\left(x_{0}, y_{0}\right)=(0,0)$ correspond to the values set in [4]. The Froude number is $\mathrm{Fr}=0.6$ based on unit length. The solutions are computed on a mesh containing $161 \times 41 \times 41$ grid points in the $x, y$ and $z$ direction, respectively, with $z=0$ as initial estimate for the FS. The correction to the FS which results from the solution of the first NavierStokes subproblem is shown in Fig. 1. The convergence behaviour of the FS 


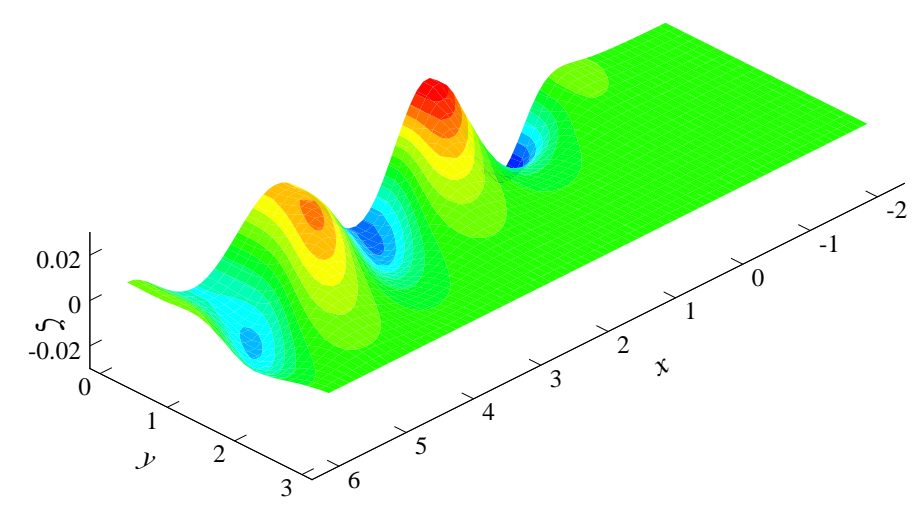

Fig. 1. FS for pressure-perturbation problem, after solving first Navier-Stokes subproblem

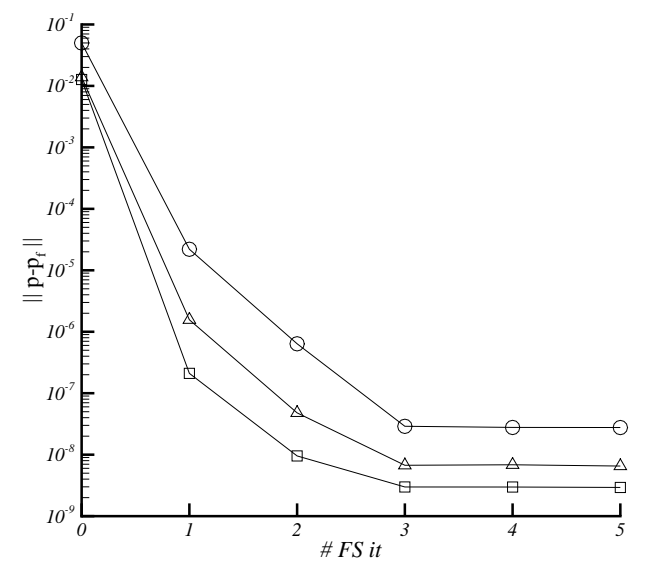

Fig. 2. FS pressure defect measured in $\|\cdot\|_{\infty}(\circ),\|\cdot\|_{1}(\square)$ and $\|\cdot\|_{2}(\triangle)$ 
iteration process, described in the previous section, is shown in Fig. 2. Correspondence of the computed solution with that from [4] is good although small differences in amplitude and wave length are present. Increasing the amplitude of the perturbation, entering the range of the mildly nonlinear wave systems, shows a reduction of the convergence rate. Computing highly nonlinear waves is not yet possible with the FS iteration method due to robustness problems of the Navier-Stokes subproblem solver.

\subsection{Realistic Ship-Hull Form}

The computational domain for this test case contains $321 \times 121 \times 41$ points. Fr and $\operatorname{Re}$ are set at 0.316 and $10^{6}$, respectively, both based on the length of the ship hull. For the initial $\Gamma_{\text {FS }}$ we take $z=0$. The wave pattern of the first Navier-Stokes subproblem, obtained after 255 iterations, is shown in Fig. 3. A comparison of this pattern with the experimental data from [5] is given in Fig. 4. For this test case no FS updates were computed yet.

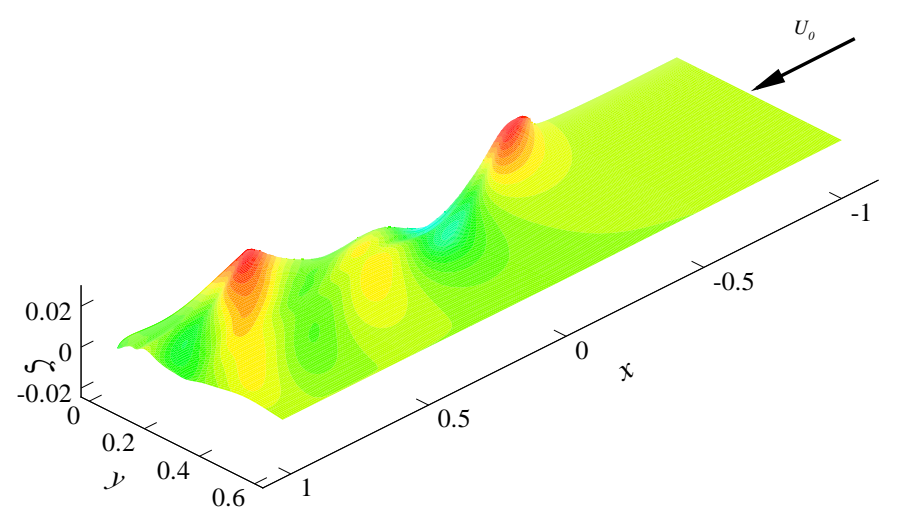

Fig. 3. Wave pattern Series 60 ship hull at $\mathrm{Fr}=0.316$ after solving first Navier-Stokes subproblem. The flow enters the domain at $x=-1$ with speed $U_{0}$. The hull is located at $-0.5 \leq x \leq 0.5$ 

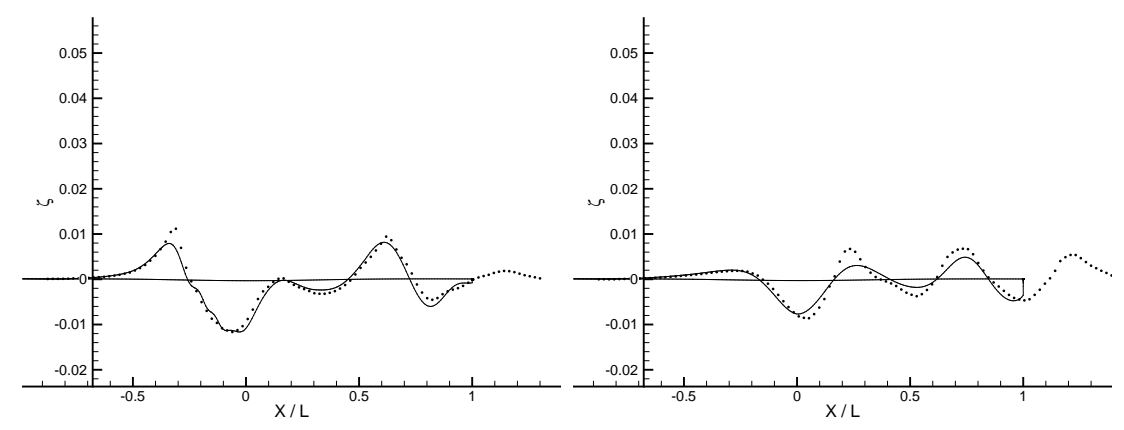

Fig. 4. Comparison of computed longitudinal wave cuts (-) with experimental results (..), Series 60 hull at $\mathrm{Fr}=0.316$ and $\mathrm{Re}=10^{6}$. Left graph : $\frac{y}{\ell}=0.0755$, right graph $: \frac{y}{\ell}=0.2067, \frac{y}{\ell}=0$ corresponds to the ship's plane of symmetry

\section{Conclusions}

The proposed FS iteration method, employing the QFSC, shows very fast convergence in the case of the pressure perturbations for linear to mildly nonlinear wave systems. For both the pressure-perturbation problem and the ship-hull problem, the solution of the first Navier-Stokes subproblem already reveals a large portion of the final wave system. The present method does not suffer from the slow transient effects encountered in time-dependent formulations. So, based on the results of the two test cases considered here, we can conclude that the new formulation of the FS flow problem possesses the proper 3D wave physics and shows very fast convergence.

\section{Acknowledgment}

This work was supported by the Dutch Technology Foundation STW.

\section{References}

1. E.H. van Brummelen, H.C. Raven, B. Koren: J. Comput. Phys. 174, 120 (2001)

2. M.J. Lighthill: Waves in Fluids (Cambridge University Press, Cambridge, 1958)

3. M. Hoekstra: Numerical Simulation of Ship Stern Flows with a Space-Marching Navier-Stokes Method. PhD Thesis, Delft University of Technology, Delft (1999)

4. D. C. Wyatt: Journal of Ship Research 44, 96 (2000)

5. Y. Toda, F. Stern, J. Longo: Report 352, Iowa Institute of Hydraulic Research, Iowa city (1991) 